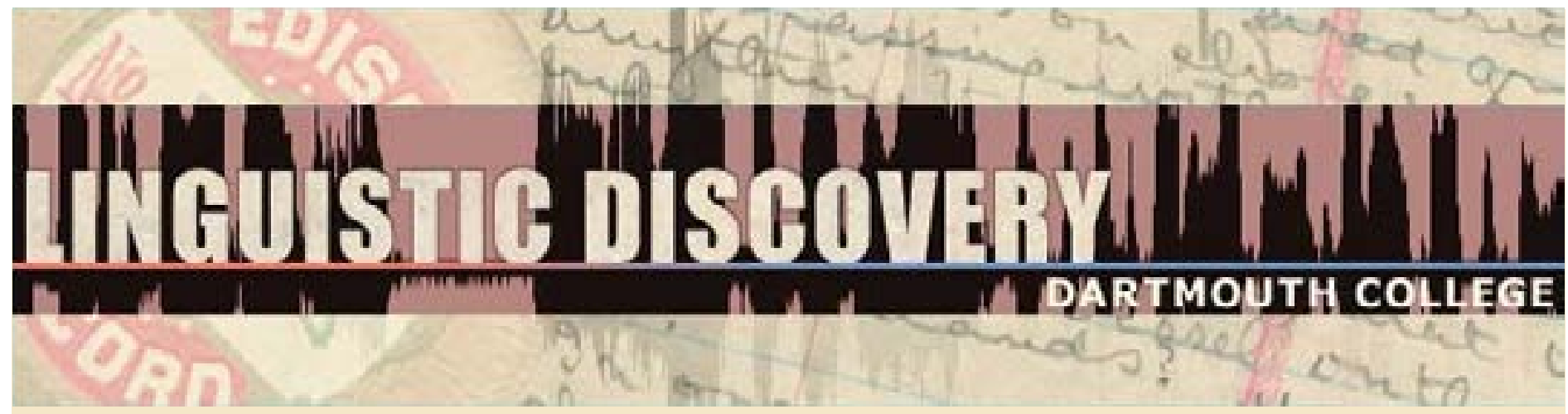

\begin{tabular}{|l|}
\hline Volume 7 \\
Issue 1 \\
2009 \\
\hline
\end{tabular}

\title{
Mono Digital Wordlist: Presentation Form
}

Kenneth S. Olson

SIL International and University of North Dakota

Mbakuwuse Tshangbaita

Communauté Évangélique du Christ en Ubangi

doi: 10.1349/PS1.1537-0852.A.343

url: http://journals.dartmouth.edu/cgi-bin/WebObjects/ Journals.woa/1/xmlpage/1/article/343

Linguistic Discovery

Published by the Dartmouth College Library Copyright to this article is held by the authors. ISSN 1537-0852 linguistic-discovery.dartmouth.edu 


\title{
Mono Digital Wordlist: Presentation Form
}

\author{
Kenneth S. Olson
}

SIL International and University of North Dakota

\author{
Mbakuwuse Tshangbaita \\ Communauté Évangélique du Christ en Ubangi
}

This paper presents a 204-item digital wordlist of Mono, an Ubangian language spoken in the Democratic Republic of the Congo. ${ }^{1}$ The wordlist includes orthographic and broad phonetic transcriptions of each word, French and English glosses, an individual WAV recording of each item, GIF images of the original field transcriptions, and metadata for resource discovery. An archival form of the wordlist was deposited into an institutional archive (the SIL Language and Culture Archives) and includes the original WAV digital recording, descriptive markup encoding of the wordlist in XML employing Unicode 5.1 transcription, TIFF images of the original field transcriptions, and the metadata record. The presentation form was then generated directly from the archival form.

\section{Introduction}

This paper presents a 204-item digital wordlist of core vocabulary in the Bili dialect of Mono, a language spoken by about 65,000 people in the northwestern part of the Democratic Republic of the Congo (ISO 639-3 code: [mnh], Lewis 2009). This presentation form was generated from an archival form of the data. The procedure we followed for creating both forms is detailed in Simons et al. (2007) and summarized below.

In addition to a description of the primary data in the form of phonetic transcription, we provide a documentation of the data in the form of digital audio recordings (cf. Himmelmann 1998, Himmelmann 2006:17-25), enabling the reader to verify and critique our transcription. This is important for this particular wordlist because Mono has some unusual phonological phenomena, including a phonemic bilabial flap $/ \gamma^{/ 2}$ (Olson and Hajek 1999, 2003, 2004); an eight-vowel system with fewer front vowels than back vowels /i, e, i, , , a, u, o, o/; labialization and palatalization realized as [o] and [e्n], respectively, that can accompany (among other segments) $/ \mathrm{h} /$ and $/ \mathrm{R} /$; echo vowel epenthesis causing a /CLV/ $(\mathrm{L}=$ liquid) underlying syllable pattern to be realized as $\left[\mathrm{CV}_{1} \mathrm{LV}_{1}\right]$; prothetic augmentation of subminimal nominal roots which, when combined with echo vowel epenthesis, leads to overapplication resulting in a non-surfaceapparent opacity effect $/ \mathrm{CLV}_{1} / \rightarrow\left[\mathrm{V}_{1} \mathrm{CV}_{1} \mathrm{LV}_{1}\right]$; and leftward vowel feature spreading subject to implicational restrictions (Olson 2005).

The materials included in this presentation of the data include the following:

\footnotetext{
${ }^{1}$ We wish to thank Judy Kuntz, Roger Olson, and Gary Simons for technical and logistic assistance. The fieldwork for this study was conducted under the auspices of SIL International and by invitation of the Communauté Évangélique du Christ en Ubangi. An earlier version of this paper was presented at the Annual Conference on African Linguistics, Athens, Georgia, April 2008.

${ }^{2}$ The right-hook v symbol $\langle\checkmark\rangle$ was added to Unicode version 5.1 at code point U+2C71. Proper rendering of the symbol in the HTML edition of this paper requires the Doulos SIL font version 4.104 or later: [http://scripts.sil.org/DoulosSILfont].
} 
- Wordlist: the present report, containing for each word a French and an English gloss, orthographic and broad phonetic transcriptions, and pertinent notes.

- Recordings: $\mathrm{WAV}^{3}$ digital recordings of each item, accessible by clicking on the orthographic form of each word in the list below. Your web browser will attempt to play the recording with the sound program that is set up as the default WAV player on your system. On Windows computers, you can download the file in the HTML version of the paper by right-clicking on the icon and selecting "Save Target As..." from the pull-down menu.

- Field transcriptions: GIF images of the field transcriptions of the data.

- Metadata: a description of the data, useful for resource discovery.

The original wordlist materials included two items: a two-page paper wordlist and a 12minute audio cassette recording. The paper wordlist presented the standardized wordlist of 204 items in French from Moñino (1988), along with the elicited Mono forms. The first author translated the wordlist into Mono with assistance from the second author (the language resource person), employing a broad phonetic transcription based on the International Phonetic Alphabet (IPA 1999).

The wordlist was recorded on September 26, 1994 in Bili, Équateur Province, Democratic Republic of the Congo. The recording was made with a Marantz PMD 420 analog cassette recorder and a Shure dynamic microphone. The language resource person was an adult male mother-tongue speaker of Mono, 35 years old at the time of the recording. During the recording session, the subject read from the paper wordlist and produced each target word once (cf. Olson 2005:121).

The recording was digitized at the International Linguistics Center (ILC) in Dallas, Texas on March 9, 2005 by Roger E. Olson. The audio cassette was played on a Marantz PMD 221 analog cassette recorder, and the recording was digitized onto a standard Windows XP computer using a Tascam US-122 USB Audio/Midi interface and CoolEdit 2000 for audio capture.

The recording was digitized at a $48 \mathrm{kHz}$ sampling rate and a 24-bit word length, which according to IASA-TC03 $(2005: 8)$ is the minimum recommended digital resolution for the archiving of analog originals. (Plichta and Kornbluh 2002 recommend $96 \mathrm{kHz}$ and 24 bits.) The recordings were stored in non-compressed WAV format, also the recommended industry standard (IASA-TC03 2005:8).

At present, the hardware of many computers does not handle $48 \mathrm{kHz}, 24$-bit audio. For the purpose of the presentation form, then, the audio recording was downsampled using CoolEdit 2000 to $44.1 \mathrm{kHz}, 16$-bit (i.e. standard audio CD quality) applying a low-pass pre-filter to the data in order to prevent aliasing (Ladefoged 1996:139-140). This sampling rate is sufficient for technical purposes since it covers nearly all acoustic information pertinent to language (Ladefoged 2003:18,26).

The two-page paper wordlist was digitized on May 14, 2008 by the first author on an HP Scanjet 4070 Photosmart Scanner at archival quality (8-bit grayscale, $300 \mathrm{dpi}$, saved as

\footnotetext{
${ }^{3}$ Definitions of WAV, XML, XSLT, and other pertinent terms employed here are available at [http://emeld.org/school/glossary.html].
} 
uncompressed TIFF files, cf. MATRIX 2001). These files were then converted to 96 dpi interlaced GIF files for the presentation form using Adobe Photoshop Elements 3.0.

The first author produced an English gloss of each item from the original French, generated an orthographic transcription from the original phonetic transcription, modified the phonetic transcription to bring it in line with the extant version of the IPA (IPA 2006), and added clarifying notes to certain entries. These data were entered into a Microsoft Excel spreadsheet using Unicode 5.1 characters [http://www.unicode.org], converted to a comma-delimited (CSV) file, and imported into TableTrans v. 1.2 software (Bird et al. 2002), where they were timealigned to the original audio recording by Judy Kuntz at the ILC on November 12-13, 2007. This annotation was outputted to an XML annotation graph output [http://www.w3.org/XML/] and transformed into an XML descriptive wordlist format using an XSLT script.

The original digitized WAV file, the digitized TIFF field transcription, the XML descriptive wordlist, and a metadata record constitute the archival form of the wordlist (Olson 2008). The metadata record follows the standard set up by the Open Language Archives Community (OLAC) [http://www.language-archives.org/OLAC/metadata.html]. ${ }^{4} \mathrm{~A}$ copy of the archival materials can be ordered on CD-ROM for a nominal fee from:

SIL Language and Culture Archives

7500 W Camp Wisdom Rd

Dallas, TX 75236 USA

archive_dallas@sil.org

The presentation form of the wordlist was then generated from the archival form. An XSLT script was employed to convert the archival XML descriptive wordlist into an HTML presentation wordlist. Then, TableTrans was used to automatically create individual sound files corresponding to each of the segments identified in the transcription process for use in the presentation form.

The data in this wordlist include certain limitations. First, there is no spoken prompt on the recording for each item. A prompt would make clear on the recording the meaning of each individual item, which could be important if the recording were separated from the annotations and descriptive markup. Second, the items are spoken in isolation rather than in carrier sentences. A carrier sentence might help avoid list intonation and make it easier to measure the length of some sounds (e.g. word-initial stops) (cf. Ladefoged 2003:7-8). Third, each word was produced only once. Recording two or three tokens of each word could help ensure that there were no mispronunciations (cf. items 45, 55, and 181). Fourth, only one speaker was recorded. Recording a larger number of speakers could help ensure that the data reflect the language as a whole and not an individual's idiolect. Ladefoged (2003:14) recommends recording half a dozen speakers of each sex. Finally, the recording was made with a dynamic microphone, whereas condenser microphones typically provide a better frequency range (cf. Ladefoged 2003:19).

\footnotetext{
${ }^{4}$ An alternative standard for linguistic metadata has been proposed called the ISLE Meta Data Initiative. See [http://www.mpi.nl/IMDI/] for more details.
} 


\section{Wordlist}

See complete resource description (i.e. metadata).

See field transcriptions:

- Page 1 (items 1-99)

- $\underline{\text { Page } 2}$ (items 100-204)

Download XML descriptive markup of the wordlist.

\begin{tabular}{|c|c|c|c|c|c|}
\hline $\begin{array}{l}\text { Item } \\
1 .\end{array}$ & $\begin{array}{l}\text { French gloss } \\
\text { abeille }\end{array}$ & $\begin{array}{l}\text { English gloss } \\
\text { bee }\end{array}$ & $\begin{array}{l}\text { Orthography } \\
\underline{\text { waterœ }}\end{array}$ & $\begin{array}{l}\text { IPA } \\
\text { [wátèrò] }\end{array}$ & Notes \\
\hline 2. & acide (vb) & tart & $\underline{\mathrm{ikpi}}$ & [íkpí] & adjective \\
\hline 3. & aile & wing & $\underline{\text { ombo }}$ & {$[\bar{o} \mathrm{mbō}]$} & \\
\hline 4. & aller & go & $\underline{\text { kœna }}$ & [kónà] & \\
\hline 5. & amer (vb) & be bitter & $\underline{\text { kœshu }}$ & [kúfù] & \\
\hline 6. & animal & animal & $\underline{\text { agya }}$ & [àgeña & \\
\hline 7. & année & year & $\underline{\text { ungu }}$ & [úngú] & \\
\hline 8. & appeler & call & $\underline{\text { kœ'e }}$ & [kéPè] & \\
\hline 9. & arbre & tree & oyo & {$[\bar{\jmath} \mathrm{j} \bar{\partial}]$} & \\
\hline 10. & attacher; lier & attach; tie & $\underline{\text { kœ'i }}$ & [kíìi] & \\
\hline 11. & blanc & white & $\underline{\mathrm{imbi}}$ & [ímbí] & \\
\hline 12. & boire & drink & $\underline{\text { kœndjo }}$ & [kóndzò] & \\
\hline \multirow[t]{2}{*}{13.} & bon (être) & good & $\underline{\text { otsho }}$ & [óţó] & adjective \\
\hline & & & $\underline{\text { kœga }}$ & [kógà] & verb \\
\hline 14. & bouche & mouth & $\underline{\mathrm{ama}}$ & [àmà] & \\
\hline 15. & bras/main & arm/hand & $\underline{\text { kane }}$ & [kāné] & \\
\hline 16. & brouillard & mist; fog & $\underline{\text { ondoro }}$ & [ōndōrō] & \\
\hline 17. & brûler (intr.) & burn (intr.) & $\underline{\text { kœju }}$ & [kúzù] & \\
\hline \multirow[t]{2}{*}{18.} & brûler (trans.) & burn (tr.) & $\underline{\text { kœsho }}$ & [kófò] & \\
\hline & & & $\underline{\text { kœsho owo }}$ & [kófò òwò] & \\
\hline 19. & ceci & this & $\underline{\text { asœe }}$ & [ásá] & \\
\hline 20. & celà & that & $\underline{\text { asœ 'ba'e }}$ & [ásó 6á?ē] & location \\
\hline 21. & ce (en question) & this & & & no entry \\
\hline 22. & cendres & ash & vorowo & [vōrōwò] & \\
\hline 23. & champ & garden & $\underline{\text { kindi }}$ & {$[\mathrm{k} \overline{\mathrm{n}} \mathrm{nd} \overline{\mathrm{f}}]$} & \\
\hline 24. & charbon & charcoal & $\underline{\text { ngiriwo }}$ & [yḡ̄̄r̄̄iwò] & \\
\hline \multirow[t]{2}{*}{25.} & chaud/chauffer & hot $/$ heat & $\underline{\text { kœwo }}$ & [kówò] & verb \\
\hline & & & owo & [òwò] & adjective \\
\hline 26. & chemin & path & $\underline{\text { awa }}$ & {$[\bar{a} w \bar{a}]$} & \\
\hline 27. & chèvre & goat & yaburu & [jābùrù] & \\
\hline 28. & chien & $\operatorname{dog}$ & yavoro & [jāvóró] & \\
\hline
\end{tabular}




\begin{tabular}{|c|c|c|c|c|c|}
\hline 29. & chose & thing & œrœ & [ว̀rò] & \\
\hline 30. & $\operatorname{cinq}$ & five & $\underline{\operatorname{mindu}}$ & [mīndû:] & \\
\hline \multirow[t]{2}{*}{31.} & cœur & heart & $\underline{\text { ulu }}$ & [ùlù] & the organ \\
\hline & & & $\underline{\text { losu }}$ & [lòsù] & figurative \\
\hline \multirow[t]{2}{*}{32.} & colline/termitière & hill & $\underline{\text { kaga }}$ & [kàgà] & mountain; large hill \\
\hline & & & $\underline{\text { koto }}$ & [kòtò] & small hill \\
\hline 33. & compter & count & $\underline{\text { kœdi }}$ & [kídì] & \\
\hline 34. & connaître & know & $\underline{\text { kœwu sœ }}$ & [kúwù sá] & \\
\hline 35. & corde & rope & $\underline{\text { uwu }}$ & [úwú] & \\
\hline 36. & corne & horn & $\underline{\dot{d d i}}$ & [ì̀'̀̀ $]$ & \\
\hline \multirow[t]{2}{*}{37.} & cou/gorge & neck/throat & $\underline{\dot{i g} \dot{i}}$ & {$[\overline{\mathrm{z}} \mathrm{g} \overline{\mathrm{f}}]$} & neck \\
\hline & & & angoro & [àngóró] & throat \\
\hline 38. & couper (couteau) & cut & $\underline{\text { kœwa }}$ & [kówà] & \\
\hline \multirow[t]{4}{*}{39.} & couper (hache) & chop & $\underline{\text { kœsherœ }}$ & [kófèrò] & split \\
\hline & & & $\underline{\text { kogara }}$ & [kágàrà] & $\begin{array}{l}\text { cut grass; mow; } \\
\text { weed }\end{array}$ \\
\hline & & & $\underline{\text { kœdjo }}$ & [kódzò] & hoe; cut with axe \\
\hline & & & kœde & [kádè] & $\begin{array}{l}\text { chop; cut down } \\
\text { tree; cut firewood }\end{array}$ \\
\hline 40. & cracher & spit & kœtu ngushi & [kútù ygúsī] & \\
\hline 41. & creuser & dig & $\underline{\text { kœdji }}$ & [kídzì] & \\
\hline 42. & cuire & cook & $\underline{k œ f a}$ & [kófâ] & \\
\hline 43. & cuisse & thigh & $\underline{\mathrm{uku}}$ & [ūkū] & \\
\hline 44. & cul; fondement & bottom & $\underline{\text { budu }}$ & [bùdú] & \\
\hline 45. & cultiver & cultivate & $\underline{\text { kœdjo }}$ & [kónd3ò] & should be [kódzò]? \\
\hline 46. & danser & dance & $\underline{\text { kœvi }}$ & [kívì] & \\
\hline 47. & debout (être) & stand & $\underline{\text { ko'ala fo }}$ & [kóPàlà fó] & \\
\hline 48. & dent & tooth & $\underline{\mathrm{iji}}$ & {$[\overline{1} 3 \overline{1}]$} & \\
\hline 49. & deux & two & $\underline{\text { bisha }}$ & [bīè̀] & \\
\hline 50. & dire & say & $\underline{\text { kœpa }}$ & [kópà] & \\
\hline 51. & donner & give & kœza & [kózà] & \\
\hline 52. & dormir & sleep & $\underline{\text { kœlo olo }}$ & [kólò ōlō] & \\
\hline \multirow[t]{2}{*}{53.} & droite (à) & right & $\underline{\text { kuni }}$ & [kūnì $]$ & \\
\hline & & & $\underline{\text { kane yakoshe }}$ & [kāné jākō $\left.\int \bar{e}\right]$ & \\
\hline 54. & eau & water & $\underline{\text { ungu }}$ & [úngú] & \\
\hline \multirow[t]{2}{*}{55.} & écorce & bark & $\underline{\text { katshu oyo }}$ & [kót $\left.\int \overline{\mathrm{u}} \overline{\mathrm{o} j} \overline{\mathrm{D}}\right]$ & should be [kátfū \\
\hline & & & & & $\overline{\mathrm{oj}} \mathrm{j}] ?$ \\
\hline 56. & éléphant & elephant & $\underline{\text { mbala }}$ & [mbàlà] & \\
\hline 57. & enfant & child & gbolo & [gbòlò] & \\
\hline 58. & enfanter & give birth & $\underline{\mathrm{k} z u}$ & [kúzù] & \\
\hline \multirow[t]{2}{*}{59.} & enfler & swell & $\underline{\text { kœmerœ }}$ & [kómèrò] & swell \\
\hline & & & kœbe & [kébè] & become fat \\
\hline
\end{tabular}




\begin{tabular}{|c|c|c|c|c|c|}
\hline 60. & entendre & hear & $\underline{\text { kœdji }}$ & [kódzì] & \\
\hline 61. & envoyer & send & kœvwa & [kóv̛à] & \\
\hline 62. & épine & thorn & $\underline{\text { ishi }}$ & [ì $\hat{\jmath}]$ & \\
\hline 63. & étoile & star & $\underline{\text { angerepe }}$ & [àngérépè] & \\
\hline 64. & étranger & stranger & gene & [gènè] & \\
\hline 65. & excréments & excrement & $\underline{\text { uyu }}$ & {$[\bar{u} j \mathrm{u}]$} & \\
\hline 66. & façonner (pot) & make (clay pot) & $\underline{\text { kœmbœrœ losu }}$ & [kómbə̀rò lōsú] & \\
\hline 67. & fagot & firewood & yawo & [jāwò] & \\
\hline 68. & faim & hunger & $\underline{\operatorname{ogo}}$ & [ògò] & \\
\hline 69. & farine & flour & $\underline{\text { zuwa }}$ & [zúwā] & \\
\hline 70. & femme & female & yashe & {$\left[j \bar{a} \int \overline{\mathrm{e}}\right]$} & \\
\hline \multirow[t]{2}{*}{71.} & fer & iron & $\underline{\text { kowo }}$ & {$[\mathrm{kōwo}]$} & \\
\hline & & & yarako & [jārākō] & \\
\hline 72. & feu & fire & owo & [òwò] & \\
\hline 73. & feuille & leaf & $\underline{\text { kako əy๊ }}$ & [kākó ว̄jō] & \\
\hline 74. & filet & net & gbanda & [gbándà] & \\
\hline 75. & finir & finish & $\underline{\text { kœka }}$ & [kókà] & \\
\hline \multirow[t]{2}{*}{76.} & flèche & arrow & $\underline{\text { wele }}$ & [wélé] & arrow \\
\hline & & & $\underline{\text { akwara }}$ & [àkoàrà] & arrowhead \\
\hline \multirow[t]{2}{*}{77.} & foie & liver & $\underline{\mathrm{ulu}}$ & [ùlù] & heart; cf. [èbè] \\
\hline & & & & & 'liver' \\
\hline 78. & forger & forge & $\underline{\text { kœts ndawo }}$ & [kátò ndàwò] & \\
\hline 79. & frapper; battre & hit & $\underline{\text { kœda }}$ & [kódà] & \\
\hline 80. & froid (être) & cold & $\underline{\mathrm{izi}}$ & [ìż̀] & adjective \\
\hline 81. & fructifier & bear fruit & $\underline{\text { kœle }}$ & [kélè] & \\
\hline 82. & fumée & smoke & $\underline{\text { ngawo }}$ & [ygáwò] & \\
\hline \multirow[t]{2}{*}{83.} & gauche (à) & left & $\underline{\text { kane yashe }}$ & [kāné jāēē] & \\
\hline & & & gele & [gèlè] & \\
\hline 84. & genou & knee & $\underline{\text { koda }}$ & [kōdà] & \\
\hline \multirow[t]{2}{*}{85.} & graisse/huile & fat/oil & $\underline{\text { kada }}$ & [kádá] & oil \\
\hline & & & ojoro & [ōzōrōo] & fat \\
\hline 86. & grand & big & $\underline{\text { egerœ }}$ & [égéró] & \\
\hline 87. & gratter (se) & scrape & $\underline{\text { kœnguru }}$ & [kóngùrù] & \\
\hline \multirow[t]{2}{*}{88.} & griller & grill & $\underline{\mathrm{kœzO}}$ & [kózò] & \\
\hline & & & $\underline{\text { kœvoro }}$ & [kóvòrò] & \\
\hline \multirow[t]{2}{*}{89.} & guerre & war & $\underline{\text { kowo }}$ & {$[\mathrm{kōwō}]$} & large-scale war \\
\hline & & & $\underline{\text { koshe }}$ & {$\left[\mathrm{k} \overline{\mathrm{o}} \int \overline{\mathrm{e}}\right]$} & \\
\hline 90. & herbe & grass & gusu & [gūsū] & \\
\hline 91. & homme & male & yakoshe & [jākōồ] & \\
\hline 92. & hyène & hyena & $\underline{\text { avwi }}$ & [àv̦ín & \\
\hline 93. & il & he & $\underline{\text { eshe }}$ & 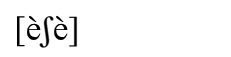 & he or she \\
\hline 94. & ils & they & œndje & [ə̀ndzēe] & \\
\hline
\end{tabular}




\begin{tabular}{|c|c|c|c|c|c|}
\hline 95. & intestins & intestines & $\underline{\text { sheta }}$ & [Sétá] & \\
\hline 96. & je & I & $\underline{\text { œmœ }}$ & [ə̄mə̄] & \\
\hline 97. & jumeaux & twins & $\underline{\text { ameya }}$ & [àméjā] & $\begin{array}{l}\text { morphology: PL- } \\
\text { meya }\end{array}$ \\
\hline 98. & lancer; jeter & throw & kœvwi & [kóv̛ì] & \\
\hline 99. & langue (organe) & tongue & $\underline{\text { tima }}$ & [tīmà] & \\
\hline 100. & laver & wash & $\underline{\text { kœyuto }}$ & [kójùtò] & \\
\hline 101. & long & long & $\underline{\text { ingiri }}$ & 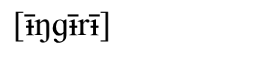 & long or tall \\
\hline 102. & lourd & heavy & $\underline{\text { kœli }}$ & [kólì] & \\
\hline 103. & lune & moon & yapu & [jápū] & \\
\hline 104. & maison & house & $\underline{\text { anda }}$ & [àndà] & \\
\hline 105. & manger & eat & $\underline{\text { kœzi }}$ & [kózì] & \\
\hline 106. & marche/marcher & walk & $\underline{\text { kœna ana }}$ & [kónà áná] & verb \\
\hline 107. & mauvais (être) & bad & ekpe & [ékpé] & adjective \\
\hline 108. & mère & mother & iya & {$[1 \mathrm{j} \overline{\mathrm{a}}]$} & \\
\hline & & & $\underline{\text { ayi }}$ & [àjīi] & \\
\hline 109. & montagne & mountain & $\underline{\text { kaga }}$ & [kàgà] & \\
\hline 110. & mordre & bite & $\underline{\text { kœlo }}$ & [kólò] & \\
\hline 111. & mortier & mortar & gafuru & [gàfūrū] & \\
\hline 112. & mouche & fly & $\underline{\text { voma }}$ & [vōmā] & \\
\hline 113. & mourir & die & $\underline{\text { kœtshu }}$ & [kútfù] & \\
\hline 114. & ne...pas & not & $\underline{\text { nene }}$ & {$[\mathrm{nēne}]$} & \\
\hline 115. & neuf; nouveau & new & $\underline{\text { tafo }}$ & [tāfò] & \\
\hline 116. & nez & nose & $\underline{\text { ngawu }}$ & [ygāwū] & \\
\hline 117. & noir & black & $\underline{\mathrm{ubu}}$ & {$[\bar{u} \mathrm{bu}]$} & \\
\hline 118. & nom & name & $\underline{\text { i'iri }}$ & {$[\overline{\mathrm{f}} \mathrm{T} \overline{\mathrm{T}} \mathrm{r} \overline{\mathrm{t}}]$} & \\
\hline 119. & nombreux (être) & be many & $\underline{\text { kœngba }}$ & [kóngbà] & \\
\hline \multirow[t]{2}{*}{120.} & nombril & navel & peya & [pējà] & "innie" \\
\hline & & & $\underline{\text { turugu }}$ & [túrúgù] & "outie" \\
\hline \multirow[t]{2}{*}{121.} & nous & we & $\underline{\mathrm{a}^{\prime} \mathrm{a}}$ & {$[\overline{\mathrm{a}} \mathrm{p} \overline{\mathrm{a}}]$} & exclusive \\
\hline & & & $\underline{\mathrm{az}}$ & [àzó] & inclusive \\
\hline 122. & nuit & night & $\underline{\text { abutsho }}$ & [àbūtfó] & \\
\hline \multirow[t]{2}{*}{123.} & œil/visage & eye/face & $\underline{\text { ala }}$ & [àlà] & eye \\
\hline & & & $\underline{\text { tshatshu }}$ & [tfátsū] & face \\
\hline 124. & œuf & egg & oporo & [ōpōrō] & \\
\hline 125. & oiseau & bird & yanu & [jānū] & \\
\hline 126. & oncle (maternel) & uncle & $\underline{a^{\prime} u}$ & [à?ú] & \\
\hline 127. & ongle/griffe & nail/claw & $\underline{\text { sungupe yokone }}$ & [sūngúpé jōk̄̄né] & \\
\hline 128. & oreille & ear & $\underline{\text { utu }}$ & {$[\bar{u} t \bar{u}]$} & \\
\hline 129. & os & bone & $\underline{\text { ngbabi }}$ & [ngbābī] & \\
\hline \multirow[t]{2}{*}{130.} & panthère & panther & gava & [gàvà] & panther \\
\hline & & & $\underline{\text { muru }}$ & [múrú] & leopard \\
\hline
\end{tabular}




\begin{tabular}{|c|c|c|c|c|}
\hline 131. peau & skin/fur & $\underline{\mathrm{oko}}$ & [ókó] & \\
\hline 132. père & father & $\underline{\mathrm{aba}}$ & [àbá] & \\
\hline 133. personne; gens & person & $\underline{\mathrm{uzu}}$ & {$[\overline{\mathrm{u} z \overline{\mathrm{u}}}]$} & \\
\hline 134. petit & small & aya & {$[\bar{a} j \bar{a}]$} & \\
\hline & & $\underline{\text { teasho }}$ & [tâ: $\left.\int \bar{o}\right]$ & \\
\hline 135. peur & fear & $\underline{\text { awa }}$ & [àwà] & \\
\hline 136. pied & foot & $\underline{\text { ada }}$ & [àdà] & \\
\hline 137. pierre/caillou & rock/pebble & $\underline{\text { badja }}$ & [bàdzà] & rock; cf. [tāmè] \\
\hline & & & & 'pebble' \\
\hline 138. plaie & wound & $\underline{\mathrm{uku}}$ & [ùkù] & \\
\hline 139. planter & plant & $\underline{\text { kœelu }}$ & [kúlù] & plant a seed \\
\hline & & $\underline{\text { kœshi }}$ & [kó $\hat{1}]$ & $\begin{array}{l}\text { plant a shoot or } \\
\text { stem }\end{array}$ \\
\hline 140. plein (être) & be full & $\underline{\mathrm{k} e s \mathrm{u}}$ & [kúsù] & \\
\hline 141. pleurer (pleurs) & cry (tears) & $\underline{\text { kœki }}$ & [ḱ̂́kì $]$ & cry \\
\hline 142. pluie & rain & yavuru & [jāvūrū] & \\
\hline 143. poil/plume & hair/feathers & $\underline{\text { usu yanu }}$ & [ùsù jānū] & feathers \\
\hline & & $\underline{\mathrm{usu}}$ & [ùsù] & hair or feathers \\
\hline 144. poisson & fish & agya tshala ngu & [àgęà tfálà ygú] & \\
\hline 145. pou & louse & $\underline{\text { atsho }}$ & [àtfó] & \\
\hline 146. poule & hen & $\underline{\text { ngoto }}$ & [nḡ̄tō] & chicken \\
\hline 147. pourri (être) & be rotten & $\underline{\text { kœfu }}$ & [kúfù] & verb \\
\hline & & $\underline{\mathrm{ufu}}$ & {$[\bar{u} f \bar{u}]$} & adjective \\
\hline 148. prendre & take & $\underline{\text { kœza }}$ & [kózà] & \\
\hline 149. profond (être) & deep & $\underline{\mathrm{ili}}$ & [ílí] & adjective \\
\hline 150. puiser & draw (water) & $\underline{\text { kœsu }}$ & [kúsù] & \\
\hline 151. quatre & four & $\underline{\text { vana }}$ & [vànā] & \\
\hline 152. queue & tail & $\underline{\text { damba }}$ & [dàmbá] & \\
\hline 153. qui? & who? & $\underline{\mathrm{e}^{\prime} \mathrm{de}}$ & [èdè] & \\
\hline 154. quoi? & what? & ga'de & [gàdè] & \\
\hline 155. racine & root & eshe эуэ & {$\left[\overline{\mathrm{e}} \int \overline{\mathrm{e}} \overline{\mathrm{o}} \mathrm{j} \overline{\mathrm{j}}\right]$} & tree root \\
\hline & & $\underline{\text { eshe }}$ & {$\left[\overline{\mathrm{e}} \int \overline{\mathrm{e}}\right]$} & root \\
\hline 156. respirer & breathe & $\underline{\text { kœwu }}$ & [kúwù] & \\
\hline 157. rester; être assis & stay; sit down & $\underline{\text { kœsœ gati }}$ & [kósò gàtí] & sit down \\
\hline 158. rire & laugh & $\underline{\text { kœmœ œmœ }}$ & [kómò ว̄m̄̄] & \\
\hline 159. rosée & dew & $\underline{\text { ngome }}$ & [ygómē] & dew \\
\hline & & $\underline{\text { ondoro }}$ & [ōndōrō] & mist \\
\hline 160. rouge & red & $\underline{\text { odoro }}$ & [ódóró] & \\
\hline 161. sable & sand & $\underline{\operatorname{mindu}}$ & {$[\min d \bar{u}]$} & \\
\hline 162. sagaie & spear & $\underline{\mathrm{udu}}$ & {$[\overline{\mathrm{u} d u}]$} & \\
\hline 163. saison des pluies & rainy season & $\underline{\text { ungu yavuru }}$ & [úngú jāvūrū] & \\
\hline 164. saison sèche & dry season & $\underline{\text { ngbugu }}$ & [ygbúgú] & \\
\hline
\end{tabular}




\begin{tabular}{|c|c|c|c|c|c|}
\hline 165. & salive & saliva & $\underline{\text { ngushi }}$ & [ygúfī] & \\
\hline 166. & sang & blood & $\underline{\text { indji }}$ & [īndzīi] & \\
\hline 167. & sec (être) & dry & $\underline{\text { o'oro }}$ & [ว̊วóró] & adjective \\
\hline 168. & sein & breast & $\underline{\text { ongo }}$ & [ว̀ngò] & \\
\hline 169. & sel & salt & $\underline{\text { ingbirit }}$ & [īngb̄̄rì & \\
\hline \multirow[t]{2}{*}{170.} & semence/graine & seed/grain & $\underline{\text { indji }}$ & [índzí] & \\
\hline & & & $\underline{\text { ele oys }}$ & [élé $\bar{j} \mathrm{j} \overline{\text { ] }}$ & \\
\hline \multirow[t]{2}{*}{171.} & sentir (intr.) & smell & $\underline{\mathrm{k} œ \mathrm{fu}}$ & [kúfù] & verb \\
\hline & & & $\underline{\mathrm{ufu}}$ & {$[\overline{\mathrm{u} f u ̄}]$} & adjective \\
\hline 172. & serpent & snake & yakoro & [jākóró] & \\
\hline 173. & soleil & sun & $\underline{\text { olo }}$ & [つ̀l’̀] & \\
\hline 174. & souffler & blow & $\underline{\text { ko'uru owo }}$ & [kúPùrù òwò] & \\
\hline 175. & sucer & suck & $\underline{\mathrm{k} e^{\prime} \mathrm{a}}$ & [kó?à] & \\
\hline 176. & sucré (être) & be sweet & $\underline{\text { kœndu }}$ & [kóndù] & \\
\hline \multirow[t]{3}{*}{177.} & termite & termite & $\underline{\text { bobo }}$ & [bòbò] & \\
\hline & & & $\underline{\text { anda abobo }}$ & [àndà àbòbò] & $\begin{array}{l}\text { termite hill; } \\
\text { morphology: àndà }\end{array}$ \\
\hline & & & & & PL-bòbò \\
\hline 178. & terre; sol & earth; soil & $\underline{\text { osho }}$ & [ว̀つว̀] & \\
\hline 179. & tête & head & kumu & [kūmù] & \\
\hline 180. & téter & nurse & $\underline{\text { kœ'a ong }}$ & [kó?à ว̀ygò] & \\
\hline 181. & tomber & fall & $\underline{\text { kœto }}$ & [kátè] & should be [kótò]? \\
\hline 182. & tortue & turtle & bakongo & [bākònḡ̄] & \\
\hline 183. & tous; tout & all & $\underline{\mathrm{k} g}$ & [kó] & \\
\hline 184. & tousser & cough & $\underline{\text { kœteks }}$ & [kótèkò] & \\
\hline 185. & tranchant (être) & be sharp & $\underline{\text { kœki }}$ & [kókì] & \\
\hline 186. & transpercer & pierce; stab & $\underline{\mathrm{k} e s \mathrm{u}}$ & [kúsù] & \\
\hline 187. & travail & work & $\underline{\text { akwa }}$ & [àkoòa & \\
\hline 188. & trois & three & $\underline{\text { voto }}$ & [vōtò] & \\
\hline \multirow[t]{2}{*}{189.} & trou & hole & $\underline{\text { kudu }}$ & [kùdú] & e.g. pit \\
\hline & & & $\underline{\text { ogoro }}$ & [ōgōrō] & e.g. hole in wall \\
\hline 190. & tu & you $(2 \mathrm{sg})$ & $\underline{œ^{\prime} b œ}$ & [ว̀bว̀] & \\
\hline 191. & tuer & kill & kœwo & [kówò] & \\
\hline 192. & un & one & $\underline{\text { bale }}$ & [bàlē] & \\
\hline 193. & urine & urine & $\underline{\text { ngindi }}$ & [ygínd̄̄] & \\
\hline \multirow[t]{2}{*}{194.} & venir & come & $\underline{\text { kœna }}$ & [kónà] & 'go' or 'come' \\
\hline & & & $\underline{\text { kœgu }}$ & [kúgù] & \\
\hline 195. & vent & wind & yugu & [jùgú] & \\
\hline 196. & ventre & abdomen & $\underline{\text { uvu }}$ & {$[\bar{u} v \bar{u}]$} & \\
\hline \multirow[t]{2}{*}{197.} & viande/chair & meat/flesh & $\underline{\text { songba }}$ & [sóngbā] & \\
\hline & & & $\underline{\text { dong } 0}$ & [dòngó] & non-starch (greens \\
\hline
\end{tabular}




\begin{tabular}{|c|c|c|c|}
\hline 198. village & village & $\underline{\text { ogo }}$ & {$[\overline{\mathrm{o} g} \overline{\mathrm{o}}]$} \\
\hline 199. voir & see & $\underline{\text { kœwu }}$ & [kúwù] \\
\hline 200. voler (oiseau) & fly & $\underline{\text { kœru }}$ & [kórù] \\
\hline 201. voler; dérober & steal & $\underline{\text { kœzi angba }}$ & [kózì āygbā] \\
\hline 202. vomir & vomit & $\underline{\text { kœndja }}$ & [kóndzà] \\
\hline 203. vouloir & want & $\underline{\text { kœyi ndœ }}$ & [kójì ndó] \\
\hline 204. vous & you $(2 \mathrm{pl})$ & e'e & {$[\overline{\mathrm{e}} \mathrm{e} \overline{\mathrm{e}}]$} \\
\hline
\end{tabular}

\section{References}

Bird, Steven; Kazuaki Maeda; Xiaoyi Ma; Haejoong Lee; Beth Randall; and Salim Zayat. 2002. TableTrans, MultiTrans, InterTrans and TreeTrans: Diverse tools built on the Annotation Graph Toolkit. Proceedings of the Third International Conference on Language Resources and Evaluation. Paris: European Language Resources Association. [http://arxiv.org/abs/cs/0204006].

Himmelmann, Nikolaus. 1998. Documentary and descriptive linguistics. Linguistics 36.161195.

Himmelmann, Nikolaus P. 2006. Language documentation: What is it and what is it good for? Essentials of language documentation, ed. by Jost Gippert, Nikolaus P. Himmelmann, and Ulrike Mosel, 1-30. Berlin: Mouton de Gruyter.

IASA-TC03. 2005. The safeguarding of the audio heritage: Ethics, principles and preservation strategy, version 3. [http://www.iasaweb.org/downloads/publications/TC03_English.pdf].

International Phonetic Association. 1999. Handbook of the International Phonetic Association. Cambridge: Cambridge University Press.

International Phonetic Association. 2006. IPA news. Journal of the International Phonetic Association 36/1.133-135.

Ladefoged, Peter. 1996. Elements of acoustic phonetics. Second edition. Chicago: University of Chicago Press.

Ladefoged, Peter. 2003. Phonetic data analysis: An introduction to fieldwork and instrumental techniques. Oxford: Blackwell.

Lewis, M. Paul. (ed.) 2009. Ethnologue: Languages of the world. 16th edition. Dallas: SIL International. [http://www.ethnologue.com].

MATRIX: The Center for Humane Arts, Letters and Social Sciences Online at Michigan State University. 2001. Digital imaging for archival preservation and online presentation: Best practices. ms. [http://www.historicalvoices.org/papers/image_digitization2.pdf].

Moñino, Yves (ed.) 1988. Lexique comparatif des langues oubanguiennes. Paris: Geuthner.

Olson, Kenneth S. 2005. The phonology of Mono (SIL International and the University of Texas at Arlington Publications in Linguistics, 140.) Dallas: SIL and UTA.

Olson, Kenneth S. 2008. Mono digital wordlist: Archival form. SIL-LCA-50695. SIL Language and Culture Archives, Dallas, Texas.

Olson, Kenneth S., and John Hajek. 1999. The phonetic status of the labial flap. Journal of the International Phonetic Association 29/2.101-114. 
Olson, Kenneth S., and John Hajek. 2003. Crosslinguistic insights on the labial flap. Linguistic Typology $7 / 2.157-186$.

Olson, Kenneth S., and John Hajek. 2004. A crosslinguistic lexicon of the labial flap. Linguistic Discovery 2/2.21-57. [http://linguistic-discovery.dartmouth.edu/cgibin/WebObjects/Journals.woa/2/xmlpage/1/article/262].

Plichta, Bartek, and Mark Kornbluh. 2002. Digitizing speech recordings for archival purposes. ms. [http://www.historicalvoices.org/papers/audio_digitization.pdf].

Simons, Gary F.; Kenneth S. Olson; and Paul Frank. 2007. Ngbugu digital wordlist: A test case for best practices in archiving and presenting language documentation. Linguistic Discovery 5/1.28-39. [http://journals.dartmouth.edu/cgi-

bin/WebObjects/Journals.woa/2/xmlpage/1/article/314]. 